\title{
Alteraciones espirométricas en adolescentes de una ciudad industrial
}

\author{
AUGUSTO RAMÍREZ', KATIA BÉJAR², ARTURO RECABARREN ${ }^{3}$ \\ 'Policlínico Los Fresnos. Cajamarca. 'Ministerio de Salud. Arequipa. \\ ${ }^{3}$ Hospital Regional III Es Salud. Arequipa.
}

\begin{abstract}
RESUMEN
OBJETIVO: Evaluar mediante espirometría a una población adolescente con edades entre 12 y 17 años residentes en Ciudad Nueva, Ilo, campamento minero con conocidos problemas de contaminación ambiental. MATERIALES Y MÉTODOS: Estudiamos 84 adolescentes -34 mujeres y 50 varones- con un espirómetro computarizado. RESULTADOS: El patrón espirométrico más frecuentemente hallado fue la obstrucción $(38,1 \%)$. Se encontró restricción en $4,8 \%$. Al correlacionar los valores encontrados con edad, talla y sexo, y compararlos con los estándares de Knudson, hallamos que CVF, VEF, PEF, y $\mathrm{FEF}_{25-75 \%}$ estaban relacionados significativamente con la estatura, pero no con edad. Existió diferencia estadísticamente significativa entre sexos, a favor del sexo nasculino, excepto para la relación VEF, /CVF. Encontramos además que la CVF y el VEF, estuvieron cercanos a lo previsto, $98,8 \%$ y $93,6 \%$, pero PEF y el FEF $25-7 \times \%$ sólo llegaron a $80,6 \%$ y $79,6 \%$. CONCLUSIONES: El patrón de alteración espirométrica más frecuente fue la obstrucción de la vía aérea. Recomendamos completar el diagnóstico a los adolescentes y ampliar el estudio a otros pobladores.
\end{abstract}

Palabras claves: Espirometría; obstrucción de las vía aérea; adolescencia.

\section{SPIROMETRIC ALTERATIONS IN ADOLESCENTS AT AN INDUSTRIAL CITY SUMMARY}

OBJECTIVE: To perform spirometry to a 12-to-17-year-old adolescent population living at Ciudad Nueva, Ilo, a city located near to a copper smelter-refinery and industrial fisheries with environmental pollution problems. MATERIAL AND METHODS: Spirometry was performed in 84 adolescents -34 women and 50 men-wich a computerized spirometer. RESULTS: The most frequent spirometric alteration was obstruction $(38,1 \%)$; restriction was found in $4,8 \%$. The Knudson's standards FVC, FEV , PEF and FEF ${ }_{25-75 \%}$ were significantly related to height, but not to age. There was statistically significant difference between genders, in favor of nien, except in FEV $/$ /FVC. FVC and FEV, values were near to those predicted $(98,8 \%$ and $93,6 \%$, respectively), but PEF and FEF ${ }_{25-75 \%}$ were only $80,6 \%$ and $79,6 \%$, respecively. CONCLUSIONS: The most frequent spirometric alteration was airway obstruction. We recommend to accomplish the clinical diagnosis in these adolescents and to study other groups of inhabitants.

Key words: Spirometry; airway obstruction; environmental pollution; adolescence.

\footnotetext{
Correspondencia:

Augusto V. Ramírez

Las Mandarimas 210

Ap. 305. La Molina. Lima 12, Perú

E-mail: averamir@usa.ner
} 


\section{INTRODUCCIÓN}

En las últimas décadas, las prucbas de función pulmonar se han convertido en instrumentos ampliamente usados para la evaluación del estado respiratorio en clínica, medicina ocupacional, medicina deportiva y salud pública $\left({ }^{1-3}\right)$. Su importancia radica en que proporcionan mediciones objetivas y reproducibles, que luego pueden ser usadas para controlar la evolución de la función respiratoria en sujetos previamente sanos o en portadores de patología. Además de valorar la respuesta al tratamiento $\left({ }^{4.5}\right)$, permiten establecer la existencia de alteraciones funcionales incipientes del sistema respiratorio, clasificar el tipo de alteración, grado de severidad y evolución $\left(^{(48)}\right.$. Las pruebas de función pulmonar proporcionan orientación sobre la naturaleza de exposición ocupacional del trabajador y el grado de deterioro de su función pulmonar $\left({ }^{9.10}\right)$. De estas pruebas, la espirometría es la más usada. Otras, como medición de volúmenes pulmonares, resistencia de vías aéreas, capacidad de difusión de monóxido de carbono y gasometría arterial, son de mayor costo y algunas tienen limitada utilidad clínica $(11.12 .13)$.

Los espirómetros actuales permiten registrar volúmenes por integración de una señal electrónica de flujo. Facilita la rapidez del cálculo el uso de programas computarizados que realizan análisis automático de las mediciones y proporcionan tablas de cifras expresadas en valor absoluto y porcentajes de los valores previstos, además de gráficos indispensables para interpretar los datos. La espirometría electrónica nos permite obtener dos tipos de registros y gráficos $\left({ }^{14}\right)$ :

- Para Tiempo Volumen: volumen espiratorio forzado en un segundo (VEF $)$, capacidad vital forzada (CVF), nujo espiratorio forzado entre $25 \%$ y $75 \%$ de la capacidad vital $\left(\mathrm{FEF}_{25-75 \%}\right)$ y el coeficiente VEF, /CVF.

- Para Flujo Volumen: pico de flujo espiratorio (PFE), FEF $25-75 \%, \mathrm{FEF}_{50 \%}, \mathrm{FEF}_{75 \%}$.

Sin embargo, es necesario puntualizar que, para obtener datos precisos y confiables, se debe cumplir normas básicas de estandarización, especialmente válidas para espirómetros digitales. Para csto, la American Thoracic Society (ATS) propuso en 1979 una serie de recomendaciones y estableció los requisitos a cumplir, tanto para el espirómetro cuanto en la ejccución de la prueba $\left({ }^{4}\right)$. En Latinoamérica, algunos países han claborado sus propias recomendaciones para interpretar la espirometría $\left({ }^{15,16}\right)$.

El examen espirométrico contribuye al diagnóstico funcional, pero no permite efectuar diagnóstico etiológico, el que sólo se puede lograr por análisis conjunto de anamnesis, examen físico, radiografía de tórax, escudio funcional cardiopulmonar y orros exámenes de laboratorio $\left(^{14-16}\right)$

Los exámenes de función pulmonar son interpretados con relación a valores de referencia, según los cuales se considera a cada sujeto como normal o anormal. Knudson, en la década de los ' 80 , confeccionó "tablas de normalidad", aplicando lo que llamó "criterios de normalidad" $\left({ }^{17}\right)$. Pero, estudios realizados en países latinos y principalmente latinoamericanos tienen problemas al aplicar estos criterios de normalidad, y los más recientes han demostrado que las tablas de Knudson, modeladas con niños anglosajones, subestiman en alrededor de $10 \%$ los valores para nuestros niños, lo que se atribuye a diferencia étnica $\left({ }^{18}\right)$.

En el Perú, hay diversos estudios espirométricos, tanto en poblaciones adultas saludables a nivel del mar y en altura, como en ciudades industriales $\left({ }^{19}\right)$, pero son pocos los que abordan el problema en infantes o adolescentes, por lo que aún continuamos aplicando estándares de otras latitudes cuando valoramos los datos espirométricos en nuestras poblaciones.

Por otro lado, es conocido que a mayor desarrollo industrial de una ciudad, mayor la contaminación que padece, lo que repercute en la salud de sus habitantes $\left({ }^{20}\right)$. Precisamente para contribuir a la investigación de este tema, planteamos nuestro estudio, que busca establecer 
el posible compromiso respiratorio de una población de adolescentes de Ciudad Nueva, Ilo, especialniente vulnerable desde el punto de vista bronquial, por las características climatológicas del poblado y la cercanía de fuentes de contaminación ambiental: entre 5 y $12 \mathrm{Km}$ se ubican dos plantas metalúrgicas primarias de cobre, refinería y fundición, que contaminan el ambiente, principalmente con anhídrido sulfuroso y en general con $\mathrm{SO}_{x}$, problema que se agrava por el material orgánico y particulado que proviene de 3 plantas industriales de harina de pescado $\left({ }^{21}\right)$. Además de estos factores de riesgo, la humedad alta en invierno y fuertes vientos polvorientos en las otras estaciones determinan un aumento de la patología pulmonar obstructiva en la población general y particularmente en niños y adolescentes $(21.22,24-26)$.

Concomitantemente, y porque el estudio de índices espirométricos -tales como CVF, VEF,

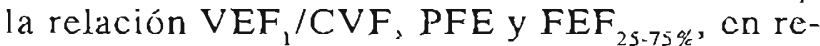
lación con las variables sexo, edad y talla- ha sido poco abordado y tampoco se ha comparado estos valores con estándares internacionales, realizamos este estudio y cotejamos los resultados con los estándares propuestos por Knudson, los cuales han sido reconocidos internacionalmente como válidos para poblaciones que carezcan de valores de referencia propios $\left({ }^{17}\right)$.

Los objetivos del estudio fueron buscar compromiso respiratorio en una población de adolescentes aparentemente sana, que vive en la zona industrial cercana a una fundición y refinería primarias de cobre y a lábricas de harina de pescado, y determinar por espirometría computarizada capacidad vital forzada, volumen espiratorio forzado al primer segundo, relación entre volumen espiratorio lorzado al primer segundo y capacidad vital forzada, pico de flujo espiratorio, flujo espiratorio forzado entre el $25 \%$ y $75 \%$ dc la capacidad vital, para relacionarlos con sexo, edad y talla. Posteriormiente, se deseó comparar los resultados con los cstándares internacionales de Knudson.

\section{MATERIALES Y MÉTODOS}

El estudio observacional y transversal lo realizamos en 110 adolescentes de 12 a 17 años de edad que asistían a un centro educativo particular secundario ubicado en el campamento minero denominado Ciudad Nueva, Ilo, en Moquegua, en el año 1999.

El estudio se realizó en Ciudad Nueva, campamento minero localizado a $3 \mathrm{Km}$ del Puerto de Ilo y a 28 msnm en el distrito de Pacocha, Departamento de Moquegua. El clima de Ilo es el de la zona costera sur, con excesiva humedad atmosférica, vientos fuertes y polvorientos, caluroso en verano, primavera y otoño, e invicrno con temperaturas bajas y precipitaciones escasas o nulas. La temperatura varía entre $14^{\circ} \mathrm{C}$ y $32^{\circ} \mathrm{C}$, con una media anual de $18^{\circ} \mathrm{C}\left({ }^{22}\right)$.

Se tomó una muestra por conveniencia seleccionando a los adolescentes de ambos scxos con edades comprendidas entre 12 y 17 años, con asistcncia regular al centro educativo, residentes en el Campamento por lo menos durante los últimos cinco años y con autorización de los padres para participar en el estudio. Se excluyó a los adolescentes portadores de patología broncopulmonar crónica, de cuadro respiratorio agudo con o sin tratamiento, o de patología torácica o abdominal (dolor torácico, cirugía rorácica o abdominal reciente, malformaciones de la columna vertebral y tórax), a los que usaban de cualquier medicamento en las últimas dos semanas y en los que se realizó una ejecución inadecuada de la prueba, a criterio de los investigadores.

Se empleó un cspirómetro Pneumocheck Wellch Allyn Modelo 61000 computarizado, de calibración automática y autocorrección para BTPS, mango portátil de diseño ergonómico, impresora térnica incorporada, con capacidad de maniobra completa para los indicadores de CV, con neumolacómetro de flujo de láminas removibles para esterilización; una balanza y un tallímetro. 
Previo a la espirometría, hicimos una entrevista personal, y un examen físico que incluyó talla y peso del examinado. Se programó al espirómetro para que registrara la curva flujo/ volumen y se le alimentó con la información de edad, talla y sexo del alumno. Para ejecutar la prueba, la investigadora explicó el procedimiento a cada participante y realizó una maniobra de capacidad vital forzada como ejemplo, para que el sujeto la imitara: 1) inspiración profunda; 2 ) colocación de una pinza nasal y cierre hermético de los labios alrededor de la boquilla; 3) soplido de todo el aire inspirado sostenida y rápidamente dentro del aparato en no menos de 3 segundos. A los participantes se les alentó a que continuaran hasta su máxima resistencia. Sc repitió el procedimiento por tres veces y se seleccionó la prueba con los mejores valores máximos. Se tomó muestras por el lapso de una semana, de las $12: 00$ a las 14:00 horas. Posteriormente, el neumólogo pediatra verilicó los trazados.

Para la descripción del grupo, usamos indicadores estadísticos de tendencia central y de dispersión: frecuencias absolutas, porcentajes, promedios y medianas. Para explicar las variables CVF, VEF, $\mathrm{FEF}_{25-75 \%}$, PEF y la relación $V E F_{1} / C V F$ en función de talla o edad, se aplicó modelos de regresión lineal simple o múltiple ( $r$ de Pearson). Como valuadores estadísticos usamos la prueba U de Mann Whitney, la prueba $Z$ para proporciones y la prueba $d c$ Kruskall Wallis ( $\left.{ }^{23}\right)$. Los valores obtenidos fueron comparados con los estándares de Knudson.

La muestra, al haber sido escogida por conveniencia, no representa a la totalidad de la población adolescente de Ciudad Nueva, Ilo. Por tanto, las conclusiones a las que se llegó sólo fueron válidas para esta población.

\section{RESULTADOS}

Del grupo inicial se excluyó a 26 estudiantes: 6 por antecedentes patológicos de asma bronquial, 3 por tiempo de residencia en Ilo menor de 5 años, 2 por problema respiratorio alto agu-
Tabla 1.- Caracteristicas de edad y sexo y frecuencia de patrones espirométricos en una población adolescente en Ciudad Nueva - Ilo, 1999.

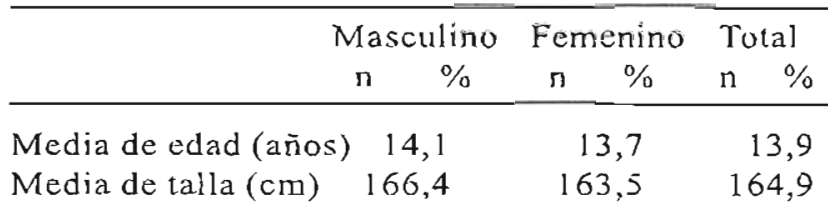

Frecuencia de alteración obstructiva*

$\mathrm{FEF}_{25-75 \%}$

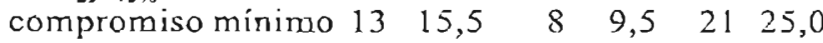
$\operatorname{VEF}_{1}(\downarrow), \operatorname{CVF}(\mathbb{N}) \quad 5 \quad 5,9 \quad 3 \quad 3,6 \quad 8 \quad 9,5$ $\begin{array}{lllllll}\operatorname{VEF}_{1}(\downarrow), \operatorname{CVF}(\downarrow) & 2 & 2,4 & \mathrm{l} & 1,2 & 3 & 3,6\end{array}$ Subtotal $\begin{array}{llllll}20 & 23,8 & 12 & 14,3 & 32 & 38,1\end{array}$

Frecuencia de alteración restrictiva ${ }^{\dagger}$

CVF (l) y $\begin{array}{lllllll}\mathrm{VEF}_{1} / \mathrm{CVF}(\mathrm{N}) & 2 & 2,4 & 2 & 2,4 & 4 & 4,8\end{array}$

$\begin{array}{lllllll}\text { Población total } & 50 & 59,6 & 34 & 40,4 & 84 & 100\end{array}$

- $X^{2}=3,53, p=0,06$

+ Prueba Exacta de Fisher $p=0,53$

do y 15 que no realizaron la prueba en forma adecuada, pese a intentar en más de 3 oportunidades. El universo se conformó con 84 adolescentes, 50 varones y 34 mujeres.

La edad promedio de la población estudiada fue 13,9 años (mediana 15 años), 14,1 años en varones y 13,7 en mujeres. La talla promedio fue $164,9 \mathrm{~cm}$ (mediana rango de 161 a $170 \mathrm{~cm}$ ), $166,4 \mathrm{~cm}$ para varones y 163,5 en mujeres. El $27,4 \%$ de la población cstudiada tuvo 15 años de edad, y en $34,5 \%$ la talla estuvo en el rango de 161 a $170 \mathrm{~cm}$ (Tabla 1).

La frecuencia de alteración obstructiva y restrictiva en la población estudiada la presentamos en la Tabla 1. Al comparar por sexos, no se cncontró diferencia significativa en cuanto a patrón restrictivo u obstructivo. Los porcentajes de los valores previstos para los índices espirométricos calculados según Knudson los mostramos en la Tabla 2. 
La Tabla 3 muestra el análisis estadístico de los parámetros estudiados y su relación con la edad. Así, en cuanto a CVF con relación a edad en varones, existió diferencia altamente significativa, mientras que en mujeres no la hubo. Para CVF relacionada con edad y sexo, existió diferencia significativa entre varones y mujeres. En la comparación entre $V E F$ y edad, en varones no existió diferencia significativa, mientras que en mujeres sí hubo diferencia y altamente significativa. La comparación entre hombres y mujeres tuvo diferencia significativa. Cuando comparamos el $\mathrm{VEF}_{1} / \mathrm{CVF}$ con edad y sexo, ni en varones ni en mujeres existió diferencia significativa; la comparación entre sexos tampoco mostró diferencia significativa. En cuanto a la relación del PEF con edad y sexo, tenemos que en varones existió diferencia significativa con relación a edad, en tanto que en mujeres no hubo diferencia significativa. Pero, al cotejar varones y mujeres, sí existió diferencia y altamente significativa. La relación del $\mathrm{FEF}_{25-75 \%}$ con edad y sexo mostró que en varones no hubo diferencia significativa, pero en mujeres la diferencia sí fue significativa. También encontramos diferencia significativa entre varones y mujeres cuando se comparó $\mathrm{FEF}_{25.75 \%}$ con la edad.

En la Tabla 4 mostramos el resultado de relacionar la talla con los índices espirométricos,
Tabla 2.- Valores previstos según estandares Knudson.

\begin{tabular}{|c|c|c|c|c|}
\hline \multirow[b]{2}{*}{ Indicador/sexo } & \multicolumn{2}{|c|}{$\begin{array}{c}\text { Correlación } \\
\text { con edad }\end{array}$} & \multicolumn{2}{|c|}{$\begin{array}{c}\text { Correlación } \\
\text { con talla }\end{array}$} \\
\hline & Masc. & Ferm. & Masc. & Fern. \\
\hline $\operatorname{CVF}(\%)$ & 98,9 & 98,8 & 98,9 & 98,8 \\
\hline $\mathrm{VEF}_{1}(\%)$ & 91,5 & 94,3 & 92,5 & 94,7 \\
\hline $\mathrm{VEF}, / \mathrm{CVF}(\%)$ & 80,7 & 79,1 & 90,7 & 79,0 \\
\hline PFE $(\%)$ & 86,8 & 74,4 & 80,9 & 78,4 \\
\hline $\mathrm{FEF}_{25-75 \%}(\%)$ & 86,8 & 74,3 & 86,8 & 74,4 \\
\hline
\end{tabular}

en el mismo orden que lo hecho para edad. Así, en lo referente a CVF con talla y sexo, tenemos que en varones existió diferencia significativa para talla. En mujeres, la diferencia fue altamente significativa. La diferencia también fue significativa cuando se contrastó varones con

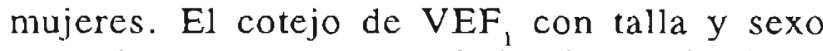
mostró que en varones existió diferencia significativa y, es más, en mujeres la diferencia fue altamente significativa. La diferencia entre sexos también fue significativa a favor de los hombres. En la relación de $V E F_{1} / C V F$ y talla vemos que ni en varones ni en mujeres existió diferencia significativa. En varones existió dife-

Tabla 3.- Pruebas de significación estadística por sexo. Îndices espirométricos por edad.

\begin{tabular}{|c|c|c|c|c|c|}
\hline & \multicolumn{2}{|c|}{ Masculino } & \multicolumn{2}{|c|}{ Femenino } & \multirow{2}{*}{$\begin{array}{l}\text { Comparación } \\
\text { entre sexos }\end{array}$} \\
\hline & Promedio $\pm \mathrm{DE}$ & Significado estad. & Promedio $\pm \mathrm{DE}$ & Significado estad. & \\
\hline $\operatorname{CVF}(\mathrm{L})$ & $3,68 \pm 0,87$ & $\mathrm{~F}: 22,6 ; p=0,002$ & 3,19 上 0,62 & $\mathrm{ZK}: 2,4 ; p=0,07$ & $\iota=2,8 ; p=0,006$ \\
\hline $\mathrm{VEF}_{1}(\mathrm{~L})$ & $2,94 \pm 0,80$ & $\mathrm{~F}: 1,7 ; p=0,18^{\circ}$ & $2,47 \pm 0,48$ & $\mathrm{~F}: 6,5 ; p=0,0003$ & $\mathrm{U}: 2,8 ; p=0,005$ \\
\hline $\mathrm{VEF}_{1} / \mathrm{CVF}(\%)$ & $80,7 \pm 10,1$ & $\mathrm{~K}: 7,0 ; p=0,13^{\circ}$ & $79,1 \pm 11,1$ & $F: 1,8 ; p=0,13^{\circ}$ & $U, Z: 0,7 ; p=0,47^{\circ}$ \\
\hline PFE $(\mathrm{L} / \mathrm{min})$ & $482 \pm 14,7$ & $\mathrm{~K}: 18,2 ; p=0,001$ & $359 \pm 11,0$ & $\mathrm{~K}: 7,7 ; p=0,10^{\circ}$ & $\mathrm{U}, \mathrm{Z}: 3,7, p=0,0002$ \\
\hline$F E F_{25-75 \%}(\mathrm{~L} / \mathrm{seg})$ & $3,03 \pm 1,15$ & $\mathrm{~K}: 18,2 ; p=0,001$ & $2,44 \pm 0,59$ & $\mathrm{~K}: 7,7 ; p=0,10^{\circ}$ & $\mathrm{U}, \mathrm{Z}: 3,2 ; p=0,007$ \\
\hline
\end{tabular}

\footnotetext{
$L / \min =$ litros por minuto

$\mathrm{L} / \mathrm{seg}=$ litros por segundo

$I=$ valor de 1 prueba de Fisher

$p=$ Valor de $p$ en cada una de las pruebas

$D E=$ Desviación estándar
}

\footnotetext{
$F=$ Valor de F en la prueba ANOVA

$U=$ Valor de $U$ en la prueba Mann Withnney

$K=$ Valor de $H$ en la prueba Kruskail Wallis

$Z=$ prueba $Z$ para proporciones

- No significativo
} 
Tabla 4.- Pruebas de significación estadistica por sexo.

indices espirometricos vs. talla.

\begin{tabular}{|c|c|c|c|c|c|}
\hline & \multicolumn{2}{|c|}{ Masculino } & \multicolumn{2}{|c|}{ Femenino } & \multirow{2}{*}{$\begin{array}{c}\text { Comparación } \\
\text { entre sexos }\end{array}$} \\
\hline & Pronsedio $\pm \mathrm{DE}$ & Significado estad. & Promedio $\pm D E$ & Significado cstad. & \\
\hline CVF (L) & $3,68 \pm 0,87$ & $F: 6,3 ; p=0,019$ & $3,19 \pm 0,62$ & $\mathrm{~F}: 23,6 ; p=0,000001$ & $l=2,8 ; p=0,006$ \\
\hline $\mathrm{VEF}_{1}(\mathrm{~L})$ & $2,97 \pm 0,80$ & $F: 18,4 ; p=0,001$ & $2,48 \pm 0,48$ & $F: 12,5 ; p=0,00009$ & $\mathrm{U}: 3,2 ; p=0,00 \mathrm{l}$ \\
\hline VEF $/$ CVF ( $(\%)$ & $90,7 \pm 10,8$ & $K: 7,0 ; p=0,13^{\circ}$ & $79,0 \pm 11,1$ & $K: 1,8 ; p=0,41^{\circ}$ & $\mathrm{U}, \mathrm{Z}: 0,7 ; p=0,47^{\circ}$ \\
\hline PFE (L/min) & $482 \pm 14,7$ & $\mathrm{~K}: 18,2 ; p=0,001$ & $359 \pm 11,0$ & $F: 5,8 ; p=0,006$ & $U, Z: 3,0 ; p=0,0002$ \\
\hline $\mathrm{FEF}_{25.75 \%}(\mathrm{~L} / \mathrm{seg})$ & $3,03 \pm 2,96$ & $\mathrm{~F}: 1,9 ; p=0,13^{\circ}$ & $2,44 \pm 0,59$ & $\mathrm{~K}: 10,2 ; p=0,0059$ & $\ell: 2,7 ; p=0,007$ \\
\hline
\end{tabular}

$L / m i n=$ litros por minulo

$\mathrm{L} / \mathrm{seg}=$ litros por segundo

$t=$ valor de $t$ prueba de Fisher

$p=$ Valor de $p$ en cada una de las pruebas

$D E=$ Desviación estándar

rencia significativa para la relación de PEF con talla y sexo. En mujeres, la diferencia fue altamente significativa. Al comparar por sexos, también hubo diferencia altamente significativa para el sexo masculino. La relación del FEF $\mathrm{FE}_{25 \%}$ con talla y sexo muestra que en varones y en mujeres hubo diferencia significativa. El contraste entre sexos también dio diferencia significativa a favor de los hombres.

Al aplicar la prueba exacta de Fisher y la de $X^{2}$ para verificar el compromiso espirométrico con relación al sexo, no encontramos diferencia significativa entre sexos.

\section{DISCUSIÓN}

Nuestro estudio investigó índices espirométricos y su relación con las variables talla, edad y sexo, en una población de estudiantes aparentemente sana, con edades entre 12 y 17 años, residentes en el campamento minero Ciudad Nueva, Ilo (28 msnm). El 100\% de los estudiados había residido en el lugar los últimos cinco años, por lo que todos provenían del mismo nivel de altitud ( $28 \mathrm{msnm}$ ).

Al analizar la variable talla, encontramos que ésta fluctuó entre 131 y $180 \mathrm{~cm}$ y que el mayor porcentaje, $34,5 \%$, estuvo en el rango de 161 -

$F=$ Valor de $F$ en la prueha ANOVA

$U=$ Valor de $U$ en la prueba Mann Wilhnney

$K=$ Valor de $H$ en la prueba Kruskail Wallis

$Z=$ prueba $Z$ para proporciones

' No significarivo

$170 \mathrm{~cm}$, que es ligeramente superior a lo hallado por Andrade y Valencia en poblaciones similares de la ciudad de Ilo $\left({ }^{22.25}\right)$.

Es consenso entre los autores aceptar cuatro tipos de patrones espirométricos básicos con las siguientes características $\left({ }^{27}\right)$ :

\begin{tabular}{|c|c|c|c|c|}
\hline Tipo & VEF & CVF & $\mathrm{VEF}_{1} / \mathrm{CVF}$ & FEF $_{25-75 \%}$ \\
\hline Restricción & $\downarrow \circ N$ & $\downarrow$ & $N \circ \uparrow$ & No $\downarrow$ \\
\hline $\begin{array}{l}\text { Obstrucción con } \\
\text { CVF normal }\end{array}$ & $\downarrow$ & $N$ & $\downarrow$ & $\mathrm{N}$ \\
\hline $\begin{array}{l}\text { Obstrucción con } \\
\text { CVF disminuida }\end{array}$ & $\downarrow$ & $\downarrow$ & $\downarrow$ & $N$ \\
\hline $\begin{array}{l}\text { Obstrucción } \\
\text { mínima }\end{array}$ & $\mathrm{N}$ & $\mathrm{N}$ & $\mathrm{N}$ & $\downarrow$ \\
\hline
\end{tabular}

$\mathrm{N}=$ normal; $\uparrow=$ aumentado, $\downarrow=$ disminuido

Para valorarlos usamos los siguientes criterios:

Alteración Restrictiva:

$\begin{array}{lll}\text { Leve } & \text { CVF: } & <\text { LIN; } \geq 65 \% \\ \text { Moderada } & \text { CVF: } & <65 \% ; \geq 50 \% \\ \text { Avanzada } & \text { CVF: } & <50 \%\end{array}$

Alteración Obstructiva:

\begin{tabular}{|c|c|c|}
\hline Mínima & $\mathrm{FEF}_{25.75 \sqrt{E}}:$ & $<\operatorname{LIN}$ \\
\hline Leve & $\mathrm{VEF}_{1}:$ & $<$ LIN; $\geq 65 \%$ \\
\hline Moderada & $V E F_{1}^{\prime}:$ & $<65 \% ; \geq 50 \%$ \\
\hline Severa & VEF: & $<50 \%$ \\
\hline
\end{tabular}




\begin{abstract}
CVF - capacidad vital forzada.
$\mathrm{FEF}_{25.75 \%}=$ flujo espiratorio forzado entre 25 y $75 \%$.

$\mathrm{VEF},=$ volumen espiratorio forzado en el primer segundo.

LIN = limite inferior de la normalidad del percentil 95.
\end{abstract}

El VEF, representa el volumen de aire que es expulsado en el primer segundo de la CVF y debe ser mayor al límite inferior de la normalidad del percentil 95. Ésta es la definición utilizada por las Comisiones de Medicina Preventiva e Invalidez ( $\left.{ }^{28}\right)$. Para otros autores, el valor del VEF, debe ser el $83 \%$ de la CVF en adultos sanos y alrededor de $90 \%$ en niños $\left({ }^{29}\right)$. En clínica, la disminución del VEF, es el índice más usado y el de mayor importancia para definir alteración obstructiva, aunque también puede estar disminuido proporcionalmente con la CVF en los problemas restrictivos $\left({ }^{30}\right)$. Nosotros hallamos que $13,1 \%$ de los examinados tenía disminución del $\mathrm{VEF}_{1}$, vale decir, encontramos $13,1 \%$ de los estudiados con patrón obstructivo, lo que concuerda con el estudio de Andrade, quien en la ciudad de llo halla $13,7 \%$ en escolares de 13 y 14 años; aunque tal vez esto no refleje su valor real, pues, al igual que nosotros, excluye a aquellos que ya tenían asma $\left({ }^{22}\right)$.

El indice de Tiffenau (relación porcentual de VEF, y CVF) también es un indicador útil, pues relaciona dos valores importantes de la espirometría, VEF, y CVF. Esta relación está disminuida en el patrón obstructivo, lo que permite el diagnóstico diferencial con el restrictivo, en el que la relación es normal o aumentada. Por otro lado, el índice de Tiffenau tiene carácter individual y es independiente de cualquier referencia o ecuación ( $\left.{ }^{12}\right)$. Encontramos esta relación alterada en $9,5 \%$, lo que corrobora el pacrón obstruccivo de esta población.

Nos referiremos aquí a ciertas incongruencias halladas en los valores del PFE, pues pese a encontrar sólo un $13,1 \%$ de la población con alteración del $\mathrm{VEF}_{1}, 69 \%$ tenía compromiso del PFE, porcentaje que nos llamó la atención, pero que no invalidó nuestro estudio, puesto que
CVF, $V_{E F}$ e indice de Tiffenau fueron normales y, como es sabido, ellos son independientes del esfuerzo realizado en la ejecución de la prueba. Además, de existir un patrón obstructivo por alteración del PFE, el mayor porcentaje debería corresponder, por "distribución normal", a alteración leve, luego moderada y por último severa $\left({ }^{23}\right)$; y porque, al aplicar nuestros criterios de exclusión, no ingresaron al estudio aquellos con problemas obstructivos confirmados. Sin embargo, esta distribución no se observó y más bien hallamos una distribución irregular: $11,9 \%$ tuvo alteración leve, $39,3 \%$ moderada y $17,9 \%$ severa. Estos hallazgos se explicarían porque, a pesar del aliento, algunos nin̄os realizaron un pobre esfuerzo al inicio de la maniobra espiratoria. Igual obstáculo halla Quadrelly en Argentina $\left({ }^{31}\right)$.

El $\mathrm{FEF}_{25.75 \%}$, también conocido como flujo espiratorio medio máximo, mide el hujo aéreo eatre los $2 / 4$ centrales de la capacidad vital forzada. En general, en síndromes obstructivos se correlaciona bien con el VEF, pero es más sensible para detectar obstrucción de lá pequeña vía aérea. El FEF 25-75\% es independiente del esfuerzo y algunos autores lo usan para definir hiperreactividad bronquial (5.12.27). La disminución del $\mathrm{FEF}_{25-75 \%}$ con CVF normal así como $\mathrm{VEF}_{1}$ y $\mathrm{VEF}_{1} / \mathrm{CVF}$ normales definen alteración obstructiva mínima $\left({ }^{12}\right)$. Individualmente, hallamos que el parámetro más frecuente de obstrucción era disminución del $\mathrm{FEF}_{25-7.5 \%}(24,9 \%)$; luego disminución del VEF, con CVF normal $(8,3 \%)$ y, por último, disminución del $\mathrm{VEF}_{1}$ con CVF disminuida $(3,6 \%)$. Para ninguno de ellos encontramos diferencia significativa al comparar por sexos.

Al sumar los indicadores de obstrucción bronquial, impresiona la cifra encontrada $38,1 \%$. Esta condición únicamente puede ser explicada por la conocida polución ambiental que soporta esta ciudad industrial, a pesar de los esfuerzos que hace la empresa minera por mitigar su parte, los gases $\mathrm{SO}_{x}\left({ }^{21.32}\right)$. Es sabido que la contaminación ambiental por agentes 
químicos y rísicos, humos o gases irritanes más materia particulada, orgánica o inorgánica, causa, entre otros problemas de salud, lesión local directa en bronquios y pulmones, o réacción alérgica, que a su vez condicionan obstrucción bronquial mínima o hiperreactividad bronquial, con manifestaciones de disfunción pulmonar $\left({ }^{3.9 .10,19.28 .33}\right)$. La fundición y refinería de llo más dos grandes fábricas de harina de pescado están localizadas entre 5 y $12 \mathrm{Km}$ al norte de Ciudad Nucva. Si bien es cierto que, principalmentc, la fundición aporta los $\mathrm{SO}_{x}$, la materia particulada es contribución de la industria pesquera. Normalmentc, el viento en Ilo corre de sur a norte, lo que teóricamente descarta la "entrada" de $\mathrm{SO}_{x}$ y materia particulada a la ciudad, pues los humos son arrastrados al desierto, ubicado al norte de llo. Sucede que varias veces al año, sobretodo en invierno, la dirección del vienco cambia y corre de norte a sur; por tanto, arrastra los contaminantes hacia las zonas pobladas. Luego, el fenómeno se complica con la humcdad, que por ese entonces casi satura el ambiente y que hace que los gases $\mathrm{SO}_{2}$ y $\mathrm{SO}_{3}$ se unan al agua y formen ácido sulfuroso y ácido sulfúrico. Esto es 10 que nos explica el aumento del patrón obstructivo en la población estudiada.

La CVF es la máxima cantidad de aire cspirado utilizando la mayor fuerza y velocidad posibles. En personas normales, la capacidad vital debe ser igual a la CVF (11.12). La CVF es útil para determinar alteraciones de tipo restrictivo, cn las que también el VEF, está disminuido proporcionalmente; por lo que la relación $\mathrm{VEF}_{1} / \mathrm{CVF}$ deviene en normal o aumentada. En nucstro estudio encontramos 4 adolescentes $(4,8 \%)$ con disminución de la CVF y por tanto con patrón restrictivo. La restricción ocurre básicamonte por dos mecanismos: o por limilación de los movimientos de la caja torácica (11.12) o por pérdida de elasticidad del tejido pulmonar, resultado de exposición ocupacional a polvos orgánicos o mineralcs, que causan la alteración restrictiva característica en la mecánica ventilatoria y en los volúmenes pulmonares $\left({ }^{10.34}\right)$. En nuestros adolescentes cstudiados, esto no podía haberse dado, pues por criterio previo excluimos la primera posibilidad; y tampoco podía ser la segunda, pues los adolescentes son escolares, no trabajan y no están expuestos a niveles elevados de polvo respirable. El carácter espirométrico limitó nuestro estudio, por lo que, al concluirlo, recomendamos a los responsables de la salud de los 4 niños que presentaron restricción ahondar la investigación, para descartar cualquier patología que pudiera estar causándola.

Al correlacionar los valores espirométricos con sexo, encontramos que la CVF en varones fue $3,68 \mathrm{~L}$ y $3,19 \mathrm{~L}$ en mujeres, $98,9 \%$ y $98,8 \%$ de los valores calculados, con diferencia significativa a favor del sexo masculino, lo cual se explica porque la capacidad vital depende del tamaño del pulmón y en general de las medidas antropométricas que, al igual que la fuerza muscular, son mayores en el hombre. Nuestros hallazgos concuerdan con Valencia, cuando estudia capacidad vital en una población de 6 a 16 años en Ilo $\left({ }^{25}\right)$.

En el análisis del VEF, con relación a edad, cncontramos una media de $2,94 \mathrm{~L}$ en varones y de $2,47 \mathrm{~L}$ en mujeres, $91,5 \%$ y $94,3 \%$ de lo cspcrado, con diferencia significativa a favor de los varones. Para el cociente VEF, y CVF con relación con edad, encontramos para varones una media de $80,7 \%$ y de $79,1 \%$ para las mujcres. No hubo diferencia significativa entre sexos, explicable porque dicha relación cs propia de cada individuo y no lienc relación con talla, edad ni sexo. En cl estudio de la relación PFE y edad encontramos para varones $482 \mathrm{~L} / \mathrm{m}$ y en mujeres $359 \mathrm{~L} / \mathrm{m}, 86,8 \%$ y $74,4 \%$ de lo esperado; la diferencia es altamente significativa a favor de los varoncs, esto debido a que PFE -igual que CVF- depende de la lortaleza de cada individuo. Para FEF ${ }_{25-75 \%}$ con relación a colad, la media fuc $3,03 \mathrm{~L} / \mathrm{s}$ cn varones y 2,44 $\mathrm{L} / \mathrm{s}$ cn mujeres, $86,8 \%$ y $74,3 \%$ de lo calculado; existió dilcrencia significativa a favor del 
varón. Los valores de la relación entre estos parámetros y edad concuerdan con lo que Corrales halla en Chile $\left({ }^{18}\right)$.

Cuando estudiamos la CVF y la relacionamos con talla, observamos que en varones la media fue 3,68 L y $3,19 \mathrm{~L}$ en mujeres, $98,9 \%$ y $98,8 \%$ de lo previsto, respectivamente, con diferencia significativa a favor de los varones. Para VEF, con relación a la talla, en varones con una media de $2,97 \mathrm{~L}$ y en mujeres con $2,48 \mathrm{~L}$, la correlación fue $92,5 \%$ y $94,7 \%$ de lo calculado, respectivamente, con diferencia significativa para los hombres. En cuanto al índice de Tiffenau con relación a talla, encontramos una media de $90,7 \%$ para varones y $79,0 \%$ en mujeres, sin diferencia significativa al comparar sexos. Para la correlación PFE con talla en varones hallamos una media en $482 \mathrm{~L} / \mathrm{m}$ y en mujeres $359 \mathrm{~L} / \mathrm{m}$ y se relacionó al $80,9 \%$ y $78,4 \%$ de lo calculado, con diferencia altamente significativa a favor de los hombres. En cuanto al $\mathrm{FEF}_{25-75 \%}$ con relación a talla, se halló medias de $3,03 \mathrm{~L} / \mathrm{s}$ en varones y $2,44 \mathrm{~L} / \mathrm{s}$ en mujeres, con $86,8 \%$ y $74,4 \%$ de lo previsto, respectivamente, y diferencia significativa a favor de los hombres. Valores similares halla Andrade cuando estudia una población de niños y adolescentes en Ilo $\left({ }^{22}\right)$.

En la comparación de edad con CVF, VEF, PFE y FEF $25.75 \%$, se halló incremento de los valores en razón directa a la edad, pero sin significado estadístico ni en varones ni en mujeres. Al comparar por sexos, se observó que para PFE sí hubo diferencia altamente significativa a favor del masculino, lo que se explica porque los hombres tienen caja torácica más ancha y mayor fuerza muscular que las mujeres $\left({ }^{3 !}\right)$. Estudios en países latinoamericanos muestran que, en mujeres, la edad tiene un papel significativo para determinar CVF, VEF, y $\mathrm{FEF}_{25-75 \%}$, mientras que en varones la edad no juega un papel significativo $\left({ }^{16.18 .35 .36}\right)$.

De igual forma, al relacionar CVF, $\mathrm{VEF}_{1}$, PFE y $\mathrm{FEF}_{25-75 \%}$ con talla, encontramos que la relación se incrementó en forma significativa al aumentar la estatura, tanto en varones como en mujeres. Este incremento fue altamente significativo para PFE a favor de los hombres; en cambio, para el índice $V_{E F_{1}} / C V F$ no se halló diferencia significativa con relación a talla ni en varones ni en mujeres, lo que puede ser explicado por la misma razón mencionada al comentar la influencia de edad. Estos resultados concuerdan con Oyarzún, quien para determinar estos índices, da mayor énfasis a la talla que a la edad $\left({ }^{16}\right)$.

Al analizar los coeficientes de correlación, observamos que los valores más altos corresponden en varones a talla contra CVF, VEF $\mathrm{FEF}_{25-75 \%}$ y PFE, mientras que en mujeres lo son sólo a talla contra CVF. En ambos sexos, el valor de los coeficientes de correlación es menor al relacionarlo con edad, por lo que concluimos con Knudson $\left({ }^{17}\right)$, Oyarzún $\left({ }^{35}\right)$ y Hernández $\left({ }^{36}\right)$ que, para la determinación de los índices espirométricos, la talla tiene mayor correlación que la edad y, por tanto, cuando se haga estudios para hallar valores de normalidad, la talla siempre debe ser incluida como variable.

En conclusión, en nuestra población estudiada, el compromiso respiratorio hallado con mayor frecuencia es el de tipo obstructivo, en un $38,1 \%$; sin embargo, se halló alteración restrictiva en $4,8 \%$. Los índices espirométricos capacidad vital forzada, volumen espiratorio forzado al primer segundo, pico de flujo espiratorio y flujo espiratorio forzado $25-75 \%$ de la capacidad vital están directa y significativamente relacionados con la estatura en ambos sexos. No ocurre lo mismo con la edad. Los hombres tienen valores más altos de capacidad vital forza$\mathrm{da}$, volumen espiratorio forzado al primer segundo, pico de flujo espiratorio y flujo espiratorio forzado $25-75 \%$ y la diferencia es estadísticamente significativa.

Por la importancia actual del tema y por 10 disperso, aislado y limitado de los estudios existentes, que no dan la potencia necesaria para la comparación estadística, sería apropiada una investigación extensa y prospectiva destinada a determinar valores propios de normalización 
para los índices espirométricos en poblaciones peruanas saludables.

\section{BIBLIOGRAFÍA}

1) Crapo R. Pulmonary function testing. N Eng J Med 1994;331(1):23-30

2) Ferris B. Epidemiology standardization project II Recommended respiratory disease questionnaires for use with adults and children in epidemiological research. Am Rev Respir Dis 1978;118:7-53.

3) Maselhom L. Pulmonary function changes in asthmatics associated with low level $\mathrm{SO}_{2}$ and $\mathrm{NO}_{2}$ air pollution, weacher, and medicine intake. Allergy 1993;48:334-8.

4) Ruppel G. Manual of Pulmonary Function Testing. USA:CV Mosby Company: 1982.

5) Issclbacher KJ. En: Harrison Principios de Medicina Interna. $13^{3}$ edición. Madrid: Interamericana Mc Graw Hill; 1994.

6) Larsson H. Valuable method for lung function measurements spirometry at ambulatory centers is excellent for early diagnosis. Lakartdinger 1995;92(47):4427-30.

7) Robbins SC. Patología y Estructura funcional. $3^{3}$ edición. México: Editoria! Interanericana; 1987.

8) Brooks SM, Weiss MA, Bernstein IL. Reactive airways dysfunction syndrome. Persistent airways hypereactivity after high level irritant exposure. Chest 1985;88:376-84.

9) Graham W. Longitudinal pulmonary function losses in Vermont granite workers. An evaluation. Chest 1994; 106(1): 125-30.

10) Ramirez AV. Efeccos de la silicosis sobre la función ventilatoria pulmonar del trabajador minero de las grandes alturas. Rev Bras Saúde Ocupacional 1990;70(18):62-9.

11) Ganong F. Tratado de Fisiologia Médica, 14;. Edición. México: Edicorial Manual Moderno. 1993.

12) Comroc JH. El Pulmón: Pruebas Funcionales. Volúmenes Pulmonares. Buenos Aires: Edlitorial Universitaria; 1963.

13) Wagner $C$, et al. The $88 \%$ saturation test: A lung function test for young child. Pediatric 1993;93(1):122-4.

14) Ancrican Thoracic Society. Lung function testing: Selection of reference values and interpretative strategies. Am Rev Respir Dis 1991;114:1202.

15) Moreno R. Recomendaciones sobre el informe espirométrico. Enf Resp Cir Torácica 1988;4:97-103.

16) Oyarzún M. Valores de referencia, criterios de interpretación e informe de espirometrías. Rev Chilena Ped \{988:59(6):400-2.

17) Knudson RJ. Change in the normal maximal expiratory flow volume curve, growth and aging. Am Rev Respir Dis $1983 ; 127: 725-34$.
18) Corrales R. Valores espirométricos normales para niños chilenos. Rev Chilena Enf Resp 1992;8:148-57.

19) Ramirez AV. Cadmium pollution in La Oroya. Peru. PAHO Bull 1986;20(4):375-9.

20) Ramirez AV, Paucar JC, Medina M. Plomo sanguíneo en habitantes de cuatro localidades peruanas. Pan Am J Public Health 1997;1(5):344-8.

21) Municipalidad Provincial de Ilo. Plan de Gestión Ambiental de la Provincia de Ilo 1999-2005. Disponible en: http://www. Receptour.com.pe/test/f/foro-porlavida/ iloplandesarrollo.htm

22) Andrade MP. Asma Bronquial: Prevalencia, Severidad y Factores Precipitantes y de Riesgo en escolares de 13-14 años de la Ciudad de Ilo. Tesis de Bachiller en Medicina Humana. UNSA. Arequipa, 1997.

23) Dawson BT. Bioestadística Médica. Interpretación de los Coeficientes de Correlación. $3^{\text {a }}$ Ed. México: Manual Moderno; 1993.

24) Farfán MF. Espirometría preoperatoria en Hidatidosis Pulmonar. Tesis de Bachiller en Medicina Humana UNSA, Arequipa; 1993.

25) Valencia VE. Capacidad Viıal en Escolares de 6 a 16 años en Cuajone e Ilo. Tesis de Bachiller en Medicina Humana UNSA. Arequipa; 1993.

26) Bejar AK. Función Ventilatoria Pulınonar en Adolescentes de 12 a 17 años de edad de un Centro Educativo del Campanento Ciudad Nueva S.P.L. Ho. Tesis de Bachiller en Medicina Humana UNSA. Arequipa; 1999.

27) Nclson J. American Thoracic Society statement. Snowbird Workshop of Standardization of Spiromerry. An Rev Res Dis 1969;110:1-195

28) Somis A. Contaminación Atmosférica. Medicina Sanitaria y Administración de Salud. Buenos Ajres: Ed. El Ateneo; 1982.

29) Gordon E, et al. Children and adult perceptions of childhood asthma. Pediatrics 1997;99:165-8.

30) Reader y Suarez G. Pruebas Funcionales Respiratorias. $2^{3}$ Ed. Argentina: El Aceneo; 1985.

31) Quadrelly S, editor. Factores de error en espirometría. Medicina (Bs As) 1994;54(1):69-81.

32) Southern Peru Copper Corporation. 1999 Annual Report. Disponible en: hup://www. southernperu.com/pages/PDF/ memoria99.pdf.

33) Rubín F. Patología: Gases Irritantes. Buenos Aires: Ed. Panamericana; 1994.

34) Zenz C, editor. Occupational Medicine, $3^{2}$ Edicion. St. Louis, USA: Mosby - Year Book Inc.; 1994.

35) Oyarzún M. Estudio funcional respiratorio. Test ventilatorios. Rev Med Chile 1983;111:862-8.

36) Hernández NR. Construcción de Tablas de valores de Pruebas Funcionales Respiratorias según Edad, Talla y Peso en niños cubanos, Revista Cubana de Pediatría 1990; 62:652-5. 